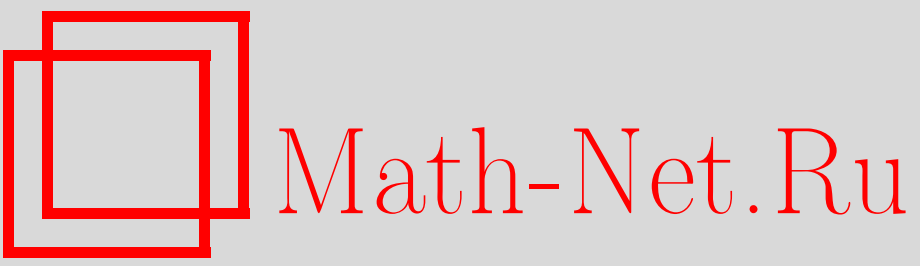

А. В. Щепетилов, Некоторые квантово-механические задачи в пространстве Лобачевского, ТМФ, 1996, том 109, номер 3, 395-405

DOI: https://doi.org/10.4213/tmf1236

Использование Общероссийского математического портала Math-Net.Ru подразумевает, что вы прочитали и согласны с пользовательским соглашением

http://www.mathnet.ru/rus/agreement

Параметры загрузки:

IP: 34.229 .108 .108

26 апреля 2023 г., 18:12:55 
ТЕОРЕТИЧЕСКАЯ

И МАТЕМАТИЧЕСКАЯ

ФИЗИКА

Том 109, № 3

декабрь, 1996

А.В. Щепетилов*

\section{НЕКОТОРЫЕ КВАНТОВО-МЕХАНИЧЕСКИЕ ЗАДАЧИ В ПРОСТРАНСТВЕ ЛОБАЧЕВСКОГО}

Рассмотрены квантово-механические задачи для потенциалов, являющихся решениями проблемы Бертрана в пространстве Лобачевского. Доказана самосопряженность соответствующих операторов Шредингера. Энергетические уровни вычислены как из уравнения Шредингера, так и методом Бора-Зоммерфельда. Обнаружен эфофект квантово-механического связывания классически инфиинитного движения. Показано, что квазиклассический предел в определенном смысле эквивалентен евклидову.

1. Как хорошо известно, среди центральных потенциалов в пространстве $\mathbf{E}^{n}$ сушествуют два выделенных (кулоновский потенциал и потенциал осциллятора), для которых все финитные траектории классической частицы замкнуты (в предположении их сушествования). Нахождение таких потенциалов и составляет содержание одной из трех разновидностей проблемы Бертрана [1]. Аналогичная задача на сфере $\mathbf{S}^{3}$ исследовалась в [2], а в пространстве Лобачевского $\mathbf{L}^{3}$ - в [3]. Было показано, что в каждом из этих случаев также сушествуют два выделенных потенциала (назовем их потенциалами типа Бертрана), для которых все финитные траектории классической частицы замкнуты. В [4] для этих потенциалов на группе $S U(2) \simeq \mathbf{S}^{3}$ была рассмотрена квантово-механическая задача.

Нашей целью является изучение квантово-механических задач с потенциалами типа Бертрана в пространствах $\mathbf{L}^{2}$ и $\mathbf{L}^{3}$. Сначала дадим, на наш взгляд, более простое, чем в $[2,3,5]$, решение проблемы Бертрана одновременно в пространствах $\mathbf{L}^{n}$ и $\mathbf{S}^{n}$ при $n \geq 2$, в котором особенно заметна схожесть этих случаев между собой и со случаем пространства $\mathbf{E}^{n}$.

Пусть $d s_{1}^{2}$ - стандартная метрика на единичной сфере $\mathbf{S}^{n-1}$. Рассмотрим следуюшие две модели односвязных пространств постоянной секционной кривизны для $n \geq 2$ :

$\mathbf{L}^{n}=\{0\} \cup(0,1) \times \mathbf{S}^{n-1}$ с метрикой

$$
d s^{2}=4 R^{2} \frac{d r^{2}+r^{2} d s_{1}^{2}}{\left(1-r^{2}\right)^{2}}, \quad r \in[0,1),
$$

и секционной кривизной $\kappa=-R^{-2}$ (модель Пуанкаре в шаре [6]);

* Московский государственный университет 


$$
\mathbf{S}^{n}=\{0, \infty\} \cup(0, \infty) \times \mathbf{S}^{n-1} \text { с метрикой }
$$

$$
d s^{2}=4 R^{2} \frac{d r^{2}+r^{2} d s_{1}^{2}}{\left(1+r^{2}\right)^{2}}, \quad r \in[0, \infty),
$$

и секционной кривизной $\kappa=R^{-2}$ (стереографическая проекция).

Функция Лагранжа точечной частицы единичной массы в центральном потенциале $U(r)$ есть $\mathcal{L}=\frac{1}{2} \dot{s}^{2}-U(r)$. Легко видеть, что, как и в евклидовом случае, движение двумерное, т. е. траектория частицы расположена на $\mathbf{L}^{2} \subset \mathbf{L}^{n}$ или $\mathbf{S}^{2} \subset \mathbf{S}^{n}$. Пусть теперь $n=2, d s_{1}=d \phi, 0 \leq \phi<2 \pi$. Введем величину

$$
\begin{array}{ll}
\xi=\frac{1}{2 R}\left(r-\frac{1}{r}\right), & -\infty \leq \xi \leq+\infty, \quad \text { для } \quad \mathrm{S}^{2}, \\
\xi=\frac{1}{2 R}\left(r+\frac{1}{r}\right), & \frac{1}{R}<\xi \leq+\infty, \quad \text { для } \quad \mathbf{L}^{2}
\end{array}
$$

и

$$
\xi=\frac{1}{r}, \quad 0<\xi \leq \infty, \quad \text { для } \quad \mathbf{E}^{2} .
$$

Тогда уравнение траектории частицы имеет вид

$$
\phi=\int M\left(2(E-U(r(\xi)))-M^{2} \xi^{2}-\kappa M^{2}\right)^{-\frac{1}{2}} d \xi
$$

где $M$ - интеграл момента, а $\kappa$ - секционная кривизна. Поскольку член $\kappa M^{2}$ можно объединить с $E$, анализ замкнутости траекторий для всех пространств проводится, как в [1] и [7] для евклидова случая, и дает возможные потенциалы $U_{1}=k \xi$ (аналог кулоновского потенциала) и $U_{2}=k \xi^{-2}$ (аналог потенциала осциллятора). Возврашаясь к более наглядной координате $r$, получим

для $\mathbf{S}^{2}$ :

$$
\begin{aligned}
& U_{1}^{s}(r)=k\left(r-\frac{1}{r}\right), \\
& U_{2}^{s}(r)=\frac{k_{1} r^{2}}{\left(r^{2}-1\right)^{2}}, \quad 0 \leq r<1 \\
& U_{2}^{s}(r)=\frac{k_{2} r^{2}}{\left(r^{2}-1\right)^{2}}, \quad 1<r \leq \infty, \\
& k_{1}, k_{2} \geq 0
\end{aligned}
$$

для $\mathbf{L}^{2}$ :

$$
U_{1}^{l}(r)=k\left(r+\frac{1}{r}\right), \quad U_{2}^{l}(r)=\frac{k r^{2}}{\left(r^{2}+1\right)^{2}} .
$$

Заметим, что особенность $U_{2}^{s}(r)$ разделяет $\mathbf{S}^{2}$ по экватору $r=1$ на области движения, которые частица не может покинуть, и этим объясняется двухпараметрический вид $U_{2}^{s}\left(k_{1}, k_{2}, r\right)$. В случае $k_{1}=k_{2} U_{2}^{s}$ можно рассматривать как потенциал в пространстве 
Римана в узком смысле - фактор-пространстве сферы $\mathbf{S}^{2}$ по центральной симметрии. В терминах расстояния $\rho$ на римановом пространстве до точки $r=0$ имеем

$$
\begin{aligned}
& U_{1}^{s}(\rho)=-\frac{k}{R} \operatorname{ctg} \frac{\rho}{R}, \quad U_{2}^{s}(\rho)=\frac{\omega^{2} R^{2}}{2} \operatorname{tg}^{2} \frac{\rho}{R}, \quad \rho=2 R \operatorname{arctg} r, \\
& U_{1}^{l}=-k R^{-1} \operatorname{cth} \frac{\rho}{R}, \quad k>0, \quad U_{2}^{l}=\frac{\omega^{2}}{2} R^{2} \operatorname{th}^{2} \frac{\rho}{R}, \quad \rho=R \ln \frac{1+r}{1-r} .
\end{aligned}
$$

Постоянные здесь подобраны так, чтобы в евклидовом пределе $R \rightarrow \infty$ получались $U_{1}^{e}=-k / \rho, U_{2}^{e}=\frac{1}{2} \omega^{2} \rho^{2}$. Отметим, что при замене $R \rightarrow i R \quad U_{j}^{s}$ переходит в $U_{j}^{l}, j=$ 1,2, и что, как и в евклидовом случае, $\left[\Delta_{3}\left(U_{1}\right)\right]=4 \pi k \delta(0)$, но в отличие от евклидова случая $\Delta_{3}\left(U_{2}^{s, l}\right) \neq$ const, где $\Delta_{3}$ - оператор Лапласа-Бельтрами в $\mathbf{L}^{3}$ или $\mathbf{S}^{3}$.

2. Рассмотрим пространства $\mathbf{L}^{2}$ и $\mathbf{L}^{3}$. Будем пользоваться моделью Бельтрами [6]: $\mathbf{L}^{n}=\{0\} \cup(0,1) \times \mathbf{S}^{n-1}$ с метрикой

$$
d s^{2}=R^{2}\left(\frac{d v^{2}}{\left(1-v^{2}\right)^{2}}+\frac{v^{2} d s_{1}^{2}}{1-v^{2}}\right), \quad v \in[0,1),
$$

$U_{1}(v) \equiv U_{1}^{l}=-k(R v)^{-1}, U_{2}(v) \equiv U_{2}^{l}=\frac{1}{2} R^{2} \omega^{2} v^{2}$. Гамильтониан частицы в $\mathbf{L}^{2}$ имеет вид

$$
H_{2}\left(p_{v}, v, p_{\phi}, \phi\right)=\frac{1}{2 R^{2}}\left(\left(1-v^{2}\right)^{2} p_{v}^{2}+\frac{1-v^{2}}{v^{2}} p_{\phi}^{2}\right)+U(v) .
$$

Рассмотрим случай $U_{1}$. Траектория частицы:

$$
v=p(1+e \cos \phi)^{-1},
$$

где

$$
p=\frac{M^{2}}{R k}, \quad M=\left|p_{\phi}\right|=\text { const }, \quad e=\sqrt{1+\frac{2 M^{2}}{k^{2}}\left(E+\frac{M^{2}}{2 R^{2}}\right)} .
$$

Условия существования движения с энергией $E$ :

$$
\begin{array}{lll}
E \geq-\frac{k^{2}}{2 M^{2}}-\frac{M^{2}}{2 R^{2}} & \text { при } & \frac{M^{2}}{R k}<1 ; \\
E>-\frac{k}{R} & \text { при } & \frac{M^{2}}{R k} \geq 1,
\end{array}
$$

а условия финитности движения:

$$
M<\sqrt{R k}, \quad E<-\frac{k}{R}
$$

Отсюда видно, что в отличие от евклидова случая при $M \geq \sqrt{R k}$ финитных движений не сушествует ни при каких $E$. Период обрашения по замкнутой орбите [3]:

$$
T=\frac{R \pi}{\sqrt{2}}\left(\left(-\frac{k}{R}-E\right)^{-\frac{1}{2}}-\left(\frac{k}{R}-E\right)^{-\frac{1}{2}}\right) .
$$


Для $U_{2}$ траектория имеет вид

$$
v^{2}=\frac{M^{2}}{R^{2}}\left(E+\frac{M^{2}}{2 R^{2}}-\sqrt{\left(E+\frac{M^{2}}{2 R^{2}}\right)^{2}-\omega^{2} M^{2}} \sin 2 \phi\right)^{-1} .
$$

Условия существования движения с энергией $E$ :

$$
\begin{array}{lll}
E \geq M \omega-\frac{M^{2}}{2 R^{2}} & \text { при } & \frac{M}{\omega R^{2}}<1 ; \\
E>\frac{\omega^{2} R^{2}}{2} & \text { при } & \frac{M}{\omega R^{2}} \geq 1,
\end{array}
$$

а условия финитности движения:

$$
\frac{M^{2}}{2 R^{2}}<E<\frac{\omega^{2} R^{2}}{2}
$$

При $M \geq \omega R^{2}$ финитных движений не существует ни при каких $E$. Период обращения по замкнутой орбите [3]: $T=2 R \pi\left(\omega^{2} R^{2}-2 E\right)^{-\frac{1}{2}}$.

3. Квантование по Бору-Зоммерфельду. Для $\mathbf{L}^{3}$ гамильтониан имеет вид

$$
\begin{aligned}
H_{3}\left(p_{v}, v, p_{\psi}, \psi, p_{\phi}, \phi\right)= & \frac{1}{2 R^{2}}\left(\left(1-v^{2}\right)^{2} p_{v}^{2}+\frac{1-v^{2}}{v^{2}}\left(\frac{p_{\phi}^{2}}{\sin ^{2} \psi}+p_{\psi}^{2}\right)\right)+U(v) \\
& 0 \leq \psi \leq \pi, \quad 0 \leq \phi \leq 2 \pi
\end{aligned}
$$

Интегралы движения: $M^{2}=p_{\psi}^{2}+p_{\phi}^{2} \sin ^{-2} \psi, L=p_{\phi}, E=H_{3}$. Базисные циклы на трехмерном торе выбираются аналогично евклидову случаю [8] и имеют те же индексы Маслова.

Рассмотрим $U_{1}$. Условия квантования имеют вид

$$
\begin{gathered}
\frac{2}{\pi \hbar} \int_{0}^{2 \phi} L d \phi=\frac{4 L}{\hbar}=4 n_{1}, \quad n_{1}=0, \pm 1, \pm 2 \ldots \\
\frac{4}{\pi \hbar} \int_{\psi_{1}}^{\psi_{2}} \sqrt{M^{2}-\frac{L^{2}}{\sin ^{2} \psi}} d \psi=\frac{4}{\hbar}(M-|L|)=2+4 n_{2}, \quad n_{2}=0,1,2 \ldots,
\end{gathered}
$$

где $\psi_{1}=\arcsin \frac{|L|}{M}, \quad \psi_{2}=\pi-\psi_{1}$,

$$
\begin{aligned}
& \frac{4}{\pi \hbar} \int_{v_{1}}^{v_{2}}\left(\frac{2 R^{2}}{\left(1-v^{2}\right)^{2}}\left(E+\frac{k}{R v}\right)-\frac{M^{2}}{\left(1-v^{2}\right) v^{2}}\right)^{\frac{1}{2}} d v= \\
& \quad=\frac{2 \sqrt{2} R}{\hbar}\left(-\sqrt{-E-\frac{k}{R}}+\sqrt{-E+\frac{k}{R}}\right)-\frac{4 M}{\hbar}=2+4 n_{3}, \quad n_{3}=0,1,2 \ldots,
\end{aligned}
$$


где $v_{1}, v_{2}$ - корни последнего подьнтегрального выражения. Отсюда получаем с учетом условий финитности движения: $L=n_{1} \hbar, M=\hbar\left(\frac{1}{2}+n_{2}+\left|n_{1}\right|\right)$,

$$
E_{n}=-\frac{n^{2} \hbar^{2}}{2 R^{2}}-\frac{k^{2}}{2 n^{2} \hbar^{2}}, \quad 1 \leq n<\frac{\sqrt{R k}}{\hbar},
$$

где $n:=1+n_{3}+n_{2}+\left|n_{1}\right|$. Кратность вырождения энергетических уровней, как и в евклидовом случае, составляет $n^{2}$. Заметим, что точно такую же формулу дает квантование по Бору, учитывающее только круговые орбиты, и что $E_{n}$ монотонно возрастает при указанных $n$.

Рассмотрим $U=U_{2}$ на $\mathbf{L}^{3}$. Отличие состоит в замене уравнения (3) условием

$$
\frac{2}{\hbar}\left(\omega R^{2}\left(1-\sqrt{1-\frac{2 E}{\omega^{2} R^{2}}}\right)-M\right)=2+4 n_{3}, \quad n_{3}=0,1,2 \ldots
$$

В итоге, подставляя $n:=2 n_{3}+n_{2}+\left|n_{1}\right|$, получим

$$
E_{n}^{(3)}=\hbar \omega\left(n+\frac{3}{2}\right)-\frac{\hbar^{2}\left(n+\frac{3}{2}\right)^{2}}{2 R^{2}}, \quad 0 \leq n<\frac{\omega R^{2}}{\hbar}-\frac{3}{2} .
$$

Кратность вырождения уровней совпадает с евклидовым случаем и составляет $(n+1)(n+2) / 2$. Аналогично для $U=U_{2}$ на $\mathbf{L}^{2}$ получаем

$$
E_{n}^{(2)}=\hbar \omega(n+1)-\frac{\hbar^{2}(n+1)^{2}}{2 R^{2}}, \quad 0 \leq n<\frac{\omega R^{2}}{\hbar}-1 .
$$

Кратность вырождения есть $n+1$. Легко видеть, что при $R \rightarrow \infty$ выражения для $E_{n}$, $E_{n}^{(3)}, E_{n}^{(2)}$ переходят в известные формулы для евклидова случая.

4. Перейдем теперь к рассмотрению соответствуюших спектральных задач для уравнения Шредингера $-\frac{\hbar^{2}}{2} \Delta \phi+U \phi=E \phi$, где $\Delta$ - оператор Лапласа-Бельтрами на $\mathbf{L}^{3}$ или $\mathbf{L}^{2}$.

4.1. Сформулируем вначале некоторые результаты о самосопряженности операторов $-\Delta+U$ в $\mathbf{L}^{n}$. Все пространства суммируемых функций понимаются относительно инвариантной меры в $\mathbf{L}^{n}$. Реализуем $\mathbf{L}^{n}$ в виде $\mathbf{R}_{+}^{n}=\left\{x \in \mathbf{R}^{n}, x^{n}>0\right\}, d s^{2}=$ $\left(x^{n}\right)^{-2} \sum_{i=1}^{n}\left(d x^{i}\right)^{2}, d \mu(x)=\left(x^{n}\right)^{-n} d x^{1} \wedge \cdots \wedge d x^{n}$,

$$
\Delta=\left(x^{n}\right)^{2} \sum_{i=1}^{n} \frac{\partial^{2}}{\left(\partial x^{i}\right)^{2}}-(n-2) x^{n} \frac{\partial}{\partial x^{n}} .
$$

Отсюда ясно видна эллиптичность оператора $\Delta$.

Пусть $V(x) \in \mathcal{L}_{\text {loc }}^{1}\left(\mathbf{L}^{n}\right), V(x) \geq 0$ - вешественнозначная функция. Определим $\hat{A}=$ $-\Delta+V(x)$ на $C_{0}^{\infty}\left(\mathbf{L}^{n}\right)$. Пусть

$$
\begin{gathered}
q(\phi, g):=(\hat{A} \phi, g)=\int_{\mathbf{R}_{+}^{n}}\left(\frac{1}{\left(x^{n}\right)^{n-2}} \sum_{i=1}^{n} \frac{\partial \phi}{\partial x^{i}} \frac{\partial \bar{g}}{\partial x^{i}}+\frac{V \phi \bar{g}}{\left(x^{n}\right)^{n}}\right) d x^{1} \wedge \cdots \wedge d x^{n}, \\
\phi, g \in C_{0}^{\infty}\left(\mathbf{L}^{n}\right) .
\end{gathered}
$$


Ясно, что $\hat{A}$ - положительный симметричный оператор на $C_{0}^{\infty}\left(\mathbf{L}^{n}\right) \subset \mathcal{L}^{2}\left(\mathbf{L}^{n}\right)$, и по теореме Х.23 из $[9$, с.200] его расширение по Фридрихсу $A$ самосопряжено. При этом $D(A) \subset D(q)$, где $D(q)=\left\{f \in \mathcal{L}^{2}\left(\mathbf{L}^{n}\right) \mid q(f, f)<\infty\right\}$.

Известные результаты [9] для евклидова случая практически полностью переносятся на случай пространств $\mathbf{L}^{n}$ (теоремы 1 и 2) и более обшо - на случай любых полных римановых пространств без края с бесконечным объемом. Единственным отличием является необходимость проведения вычислений в локальных (например, нормальных [10]) координатах.

Теорема 1. Справедлива формула

$$
\begin{gathered}
D(A)=\left\{\phi \in \mathcal{L}^{2}\left(\mathbf{L}^{n}\right) \mid V \phi \in \mathcal{L}_{\text {loc }}^{1}\left(\mathbf{L}^{n}\right),-\Delta \phi+V \phi \in \mathcal{L}^{2}\left(\mathbf{L}^{n}\right)\right\}, \\
A \phi=-\Delta \phi+V \phi .
\end{gathered}
$$

(Здесь и всюду далее производные понимаются в смысле обобщенных функций.)

ТЕорема 2. Пусть $V \in \mathcal{L}_{\mathrm{loc}}^{2}\left(\mathbf{L}^{n}\right), \quad V \geq 0$. Тогда $-\Delta+V$ самосопряжен в существенном на $C_{0}^{\infty}\left(\mathbf{L}^{n}\right)$.

Следующий вполне стандартный результат гарантирует локальную суммируемость вторых частных производных функций из $D(A)$.

TeOpema 3. Пусть $V \in \mathcal{L}_{\mathrm{loc}}^{\infty}\left(\mathbf{L}^{n}\right)$. Тогда $D(A) \subset W_{\mathrm{loc}}^{2,2}$.

ДокаЗАТЕЛЬСтво. Пусть $f \in D(A)$ и пусть $\Omega$ - область в $\mathbf{L}^{n}$ с компактным замыканием. Тогда $\left.(A f)\right|_{\Omega} \in \mathcal{L}^{2}(\Omega)$, откуда в силу [11, с.177] $\left.f\right|_{\Omega^{\prime}} \in W^{2,2}\left(\Omega^{\prime}\right), \forall \Omega^{\prime} \subset \subset \Omega$. Ввиду произвольности $\Omega$ имеем нужный результат.

Формулировка теоремы 4 переносится из [9], хотя ее доказательство несколько видоизменяется, т.к. мы не можем воспользоваться преобразованием Фурье для доказательства основной оценки. При доказательстве существенно используется транзитивность групшы изометрий $\mathbf{L}^{n}$.

ТеОрема 4. Пусть $V \in \mathcal{L}^{\infty}\left(\mathbf{L}^{3}\right)+\mathcal{L}^{2}\left(\mathbf{L}^{3}\right), \quad V$ - вещественная функиия. Тогда $A$ самосопряжен в существенном на $C_{0}^{\infty}\left(\mathbf{L}^{3}\right)$ и самосопряжен на

$$
D(-\Delta)=\left\{\phi \in \mathcal{L}^{2}\left(\mathbf{L}^{3}\right) \mid-\Delta \phi \in \mathcal{L}^{2}\left(\mathbf{L}^{3}\right\} .\right.
$$

ДокАЗАТЕЛЬСТВо. В силу теорем Х.12 и Х.15 из [9] достаточно доказать оценку: $\forall a>0 \quad \exists b>0$ такое, что $\forall \phi \in D(-\Delta)$ верно $\|\phi\|_{\infty} \leq a\|\Delta \phi\|_{2}+b\|\phi\|_{2}$, где $\|\cdot\|_{2}-$ норма в $\mathcal{L}^{2}\left(\mathbf{L}^{3}\right)$, а $\|\cdot\|_{\infty}$ - норма в $\mathcal{L}^{\infty}\left(\mathbf{L}^{3}\right)$. Пусть $\Omega \subset \subset \mathbf{L}^{3}$ - область с компактным замыканием. В соответствии с теоремой 8.24 из [11] множество

$$
P(B)=\left\{\phi \in D(-\Delta),\|\Delta \phi\|_{2, \Omega}+\|\phi\|_{2, \Omega}<B\right\}
$$

компактно вложено в $C\left(\bar{\Omega}^{\prime}\right), \forall \Omega^{\prime} \subset \subset \Omega, \forall B>0$. Здесь $\|\cdot\|_{2, \Omega}-$ норма в $\mathcal{L}^{2}(\Omega)$. Покажем, что $\forall a>0 \exists b>0$ такое, что $\forall \phi \in D(-\Delta)$ выполнено $\|\phi\|_{\mathrm{C}\left(\bar{\Omega}^{\prime}\right)} \leq a\|\Delta \phi\|_{2, \Omega}+$ $b\|\phi\|_{2, \Omega}$. Допустим противное. Это означает, что $\exists a>0,\left\{b_{n}\right\} \rightarrow \infty,\left\{\phi_{n}\right\} \subset D(-\Delta)$ 
такие, что $\left\|\phi_{n}\right\|_{\mathrm{C}\left(\bar{\Omega}^{\prime}\right)}>a\left\|\Delta \phi_{n}\right\|_{2, \Omega}+b_{n}\left\|\phi_{n}\right\|_{2, \Omega}=: \alpha_{n}$. Без ограничения обшности (при необходимости нормируя) можно считать, что $\left\|\Delta \phi_{n}\right\|_{2, \Omega}+\left\|\phi_{n}\right\|_{2, \Omega}=1$. Ввиду компактности $P(2)$ в $\mathrm{C}\left(\bar{\Omega}^{\prime}\right) \quad \exists \phi_{n_{k}} \rightarrow \psi$ в $\mathrm{C}\left(\bar{\Omega}^{\prime}\right)$, а значит, и в $\mathcal{L}^{2}\left(\Omega^{\prime}\right)$. Последовательность $\alpha_{n_{k}}$ ограничена и (т.к. $\left.b_{n_{k}} \rightarrow \infty\right)\left\|\phi_{n_{k}}\right\|_{2, \Omega} \rightarrow 0$. Но отсюда $\left\|\phi_{n_{k}}\right\|_{2, \Omega^{\prime}} \rightarrow 0, \psi=0$ и $\left\|\Delta \phi_{n_{k}}\right\|_{2, \Omega} \rightarrow 1$. Переходя к пределу, получаем противоречие: $0=\|\psi\|_{\mathrm{C}\left(\bar{\Omega}^{\prime}\right)} \geq a>0$. Таким образом, $\forall a>0 \exists b>0$ такое, что $\forall \phi \in D(-\Delta)$ выполнено

$$
\|\phi\|_{\mathrm{C}\left(\bar{\Omega}^{\prime}\right)} \leq a\|\Delta \phi\|_{2, \Omega}+b\|\phi\|_{2, \Omega} \leq a\|\Delta \phi\|_{2}+b\|\phi\|_{2} .
$$

Пусть теперь точка $x \in \mathbf{L}^{3}$ произвольна. Сушествует изометрия $\gamma$, переводящая $x$ в $x^{\prime} \in \Omega^{\prime}$. При этом

$$
\|\Delta(\phi \circ \gamma)\|_{2}=\|\Delta \phi\|_{2}, \quad\|\phi \circ \gamma\|_{2}=\|\phi\|_{2},
$$

откуда имеем

$$
\|\phi\|_{\infty} \leq a\|\Delta \phi\|_{2}+b\|\phi\|_{2}
$$

4.2. Далее мы найдем собственные значения уравнения Шредингера с потеншиалом $U_{1}$ в $\mathbf{L}^{3}$ и потенциалом $U_{2}$ в $\mathbf{L}^{3}$ и $\mathbf{L}^{2}$. Из общей теории эллиптических уравнений следует бесконечная гладкость собственных функций в точках гладкости коэффициентов уравнения и возможность их поиска методом разделения переменных в нашем центрально-симметричном случае. Рассмотрим $U=U_{1}$ в $\mathbf{L}^{3}$. Произведем замену $u:=v^{-1}$, $1<u \leq \infty$. Тогда

$$
\Delta=\frac{1}{R^{2}}\left(\left(u^{2}-1\right)^{2} \frac{\partial^{2}}{\partial u^{2}}+\left(u^{2}-1\right) \Delta_{s}\right), \quad \omega_{3}=\frac{R^{3} d u \wedge \omega_{s}^{2}}{\left(u^{2}-1\right)^{2}},
$$

где $\omega_{s}^{2}$ - форма объема на единичной сфере $\mathbf{S}_{1}^{2}$ со стандартной метрикой. Пусть $\psi=W(u) \Phi_{l, m}(x), \quad x \in \mathbf{S}_{1}^{2}, \quad \Delta_{s} \Phi_{l, m}=-l(l+1) \Phi_{l, m}$, где $\Phi_{l, m}$ - сферическая гармоника. Уравнение для $W$ имеет вид

$$
W^{\prime \prime}(u)+\frac{a+b u+c u^{2}}{\left(u^{2}-1\right)^{2}} W(u)=0,
$$

где $a=2 R^{2} E \hbar^{-2}+l(l+1), b=2 R k \hbar^{-2}, c=-l(l+1)$. Отметим, что переход к евклидову пределу осушествляется заменой $r=R / u$ и устремлением $R \rightarrow \infty$ при сохранении величины $r$, которая переходит в евклидов радиус.

Уравнение (7) является уравнением Римана [12] с тремя особыми точками $\pm 1, \infty$, в окрестности которых его решение как функция комплексного переменного ветвится. Критические показатели в этих точках имеют вид

$$
\begin{aligned}
\rho_{1}^{(1)} & =\frac{1+\sqrt{1-a-b-c}}{2}, & \rho_{2}^{(1)} & =\frac{1-\sqrt{1-a-b-c}}{2}, \\
\rho_{1}^{(-1)} & =\frac{1+\sqrt{1-a+b-c}}{2}, & \rho_{2}^{(-1)} & =\frac{1-\sqrt{1-a+b-c}}{2}, \\
\rho_{1}^{(\infty)} & =l, & \rho_{2}^{(\infty)} & =-1-l .
\end{aligned}
$$


В соответствии с теоремой 4 нас интересуют решения, для которых

$$
\int_{1}^{\infty} \frac{|W|^{2} d u}{\left(u^{2}-1\right)^{2}}<\infty \quad \text { и } \quad \Delta\left[W\left(\frac{1}{v}\right) \Phi_{l, m}(x)\right] \in \mathcal{L}^{2}\left(\mathbf{L}^{3}\right) .
$$

Первое условие в (8) исключает асимптотику при $u \rightarrow 1$, соответствуюшую $\rho_{2}^{(1)}$, причем должно быть

$$
1-a-b-c>0
$$

и асимптотику при $u \rightarrow \infty$, соответствующую $\rho_{2}^{(\infty)}$ при $l>0$. Второе же условие в $(8)$ исключает $\rho_{2}^{(\infty)}$ при $l=0$. Таким образом, собственные функции должны иметь асимптотику порядка $(u-1)^{\rho_{1}^{(1)}}$ при $u \rightarrow 1$ и $u^{-l}$ при $u \rightarrow \infty$.

Исходя из общей теории уравнения Римана [12] приведем уравнение (7) к гипергеометрическому виду заменой $y(\xi)=W(u)(u-1)^{-\rho_{1}^{(1)}}(u+1)^{-\rho_{2}^{(-1)}}, \xi=\frac{1}{2}(1-u)$. Тогда справедливо

$$
\xi(1-\xi) y^{\prime \prime}+(\gamma-\xi(\alpha+\beta+1)) y^{\prime}-\alpha \beta y=0 .
$$

Критические показатели функции $y$ следующие: $\alpha$ и $\beta$ в $\infty ; 0$ и $1-\gamma$ в $0 ; 0$ и $\gamma-\alpha-\beta$ в 1, вследствие чего получаем

$$
\begin{gathered}
\alpha=\rho_{1}^{(\infty)}+\rho_{1}^{(1)}+\rho_{2}^{(-1)}=l+1+\frac{1}{2}(\sqrt{1-a-b-c}-\sqrt{1-a+b-c}), \\
\beta=\rho_{2}^{(\infty)}+\rho_{1}^{(1)}+\rho_{2}^{(-1)}=-l+\frac{1}{2}(\sqrt{1-a-b-c}-\sqrt{1-a+b-c}), \\
1-\gamma=\rho_{2}^{(1)}-\rho_{1}^{(1)}=-\sqrt{1-a-b-c}, \\
\gamma=1+\sqrt{1-a-b-c}, \quad \alpha-\beta=2 l+1 .
\end{gathered}
$$

Нужная нам функция $y(\xi)$ должна иметь асимптотику: const в 0 и $\xi^{-\alpha}$ при $\xi \rightarrow-\infty$. Это означает, что

$$
y(\xi)=F(\alpha, \beta, \gamma, \xi) \quad \text { и } \quad \lim _{\xi \rightarrow-\infty} F(\alpha, \beta, \gamma, \xi) \xi^{\beta}=0
$$

Поскольку [13]

$$
\lim _{\xi \rightarrow-\infty} F(\alpha, \beta, \gamma, \xi) \xi^{\beta}=\frac{\Gamma(\gamma) \Gamma(\alpha-\beta)}{\Gamma(\alpha) \Gamma(\gamma-\beta)}
$$

и $\gamma>0, \alpha-\beta>0, \gamma-\beta=1+l+\frac{1}{2}(\sqrt{1-a-b-c}+\sqrt{1-a+b-c})>0$, то должно быть: $\alpha=-m+1, m=1,2,3 \ldots$, т.е.

$$
-\sqrt{1-a-b-c}+\sqrt{1-a+b-c}=2(m+l)=2 n, \quad n=1,2,3 \ldots,
$$

откуда

$$
\sqrt{1-a-b-c}+\sqrt{1-a+b-c}=\frac{b}{n}
$$


Складывая и вычитая (10) и (11), с учетом (9) получаем $\sqrt{1-a+b-c}=\frac{b}{2 n}+n$, $\sqrt{1-a-b-c}=\frac{b}{2 n}-n>0$. Отсюда, подставляя значения $a, b, c$, получим

$$
E_{n}=\frac{\hbar^{2}}{2 R^{2}}-\frac{n^{2} \hbar^{2}}{2 R^{2}}-\frac{k^{2}}{2 n^{2} \hbar^{2}}, \quad 1 \leq n<\frac{\sqrt{R k}}{\hbar} .
$$

Заметим, что при указанных значениях $n$ энергия $E_{n}$ монотонно возрастает. Ее уровни по-прежнему $n^{2}$-кратно вырожденны. Мы видим, что уравнение (12) отличается от (4) наличием дополнительного члена $\frac{\hbar^{2}}{2 R^{2}}$, который, как мы сейчас покажем, ответствен за любопытный эффект квантово-механического связывания классически инфинитного движения.

Второе условие финитности классического движения (1) может быть не выполнено для $E_{n}$ из (12), т.к., выбирая $R k$ так, что $\sqrt{R k} \hbar^{-1}=n_{1}+\varepsilon, \varepsilon>0$, можно добиться, что $E_{n_{1}}>-\frac{k}{R}=U_{1}^{\max }$, причем всегда $E_{n}-U_{1}^{\max }<\frac{\hbar^{2}}{2 R^{2}}$. Заметим, что, как и в евклидовом случае, оператор квадрата полного момента есть $-\hbar^{2} \Delta_{s}$, его собственные значения суть $\hbar^{2} l(l+1)=\hbar^{2}(n-m)(n-m+1) \leq \hbar^{2}(n-1) n<R k$, что удовлетворяет первому условию из (1).

Обратимся теперь к виду радиальной собственной функции $W_{n, l}$, отвечающей энергии $E_{n}$. При $\alpha=1-m, \beta=-m-2 l, \gamma=1+\frac{R k}{n \hbar^{2}}-n \quad y(\xi)=F(\alpha, \beta, \gamma, \xi)$ есть полином в точности $(m-1)$-й степени. Его точный вид:

$$
y(\xi)=P_{m-1}(\xi)=1+\sum_{p=1}^{m-1} \frac{(\alpha)_{p}(\beta)_{p}}{p !(\gamma)_{p}} \xi^{p}
$$

где $(\alpha)_{p}:=\alpha(\alpha+1) \ldots(\alpha+p-1)$. Поскольку $\rho_{1}^{(1)}=\frac{1}{2}\left(1+\frac{R k}{n \hbar^{2}}-n\right), \rho_{2}^{(-1)}=$ $\frac{1}{2}\left(1-\frac{R k}{n \hbar^{2}}-n\right)$, получаем

$$
W_{n, l}(u)=P_{n-l-1}\left(\frac{1-u}{2}\right)\left(u^{2}-1\right)^{\frac{1-n}{2}}\left(\frac{u-1}{u+1}\right)^{\frac{R k}{2 n \hbar^{2}}} .
$$

При переходе к евклидову пределу нужно предварительно положить $u=R / r$ и умножить $W_{n, l}$ на $R^{n-1}$, а затем устремить $R \rightarrow \infty$. При этом $r$ станет евклидовым радиуcom.

Уравнение для радиальных собственных функций для $U_{2}$ также приводится к уравнению Римана с независимой переменной $t=u^{2}$. Выпишем только результаты. Собственные значения, соответствуюшие потенциалу $U_{2}$ в $\mathbf{L}^{3}$, суть

$$
\begin{gathered}
E_{n}^{(3)}=\frac{\hbar^{2}}{2 R^{2}}\left(\frac{3}{4}-\left(n+\frac{3}{2}\right)^{2}\right)+\omega \hbar\left(n+\frac{3}{2}\right) \sqrt{1+\frac{\hbar^{2}}{4 \omega^{2} R^{4}}} \\
0 \leq n<\frac{\omega R^{2}}{\hbar} \sqrt{1+\frac{\hbar^{2}}{4 \omega^{2} R^{4}}}-\frac{3}{2}
\end{gathered}
$$

Степень вырождения энергетических уровней, как и в евклидовом случае, есть $(n+1) \times$ $(n+2) / 2$. Собственные функции, соответствующие $E_{n}^{(3)}$, имеют вид

$$
\psi_{n, l, m}(u, x)=\left(u^{2}-1\right)^{-\frac{\sigma+n}{2}} u^{\sigma} P_{n, l}^{(3)}\left(u^{2}\right) \Phi_{l, m}(x),
$$


где

$$
\begin{gathered}
\sigma=\frac{1}{2}\left(1-\sqrt{1+\frac{4 \omega^{2} R^{4}}{\hbar^{2}}}\right), \quad x \in \mathbf{S}^{2}, \\
P_{n, l}^{(3)}(t)=1+\sum_{j=1}^{k} \frac{(-k)_{j}\left(-k-l-\frac{1}{2}\right)_{j}}{j !(-\sigma-n)_{j}}(1-t)^{j}, \quad n=2 k+l .
\end{gathered}
$$

Для потенциала $U_{2}$ в $\mathbf{L}^{2}$ :

$$
\begin{gathered}
E_{n}^{(2)}=-\frac{\hbar^{2}}{2 R^{2}}(n+1)^{2}+\omega \hbar(n+1) \sqrt{1+\frac{\hbar^{2}}{4 \omega^{2} R^{4}}} \\
0 \leq n<\frac{\omega R^{2}}{\hbar} \sqrt{1+\frac{\hbar^{2}}{4 \omega^{2} R^{4}}}-1
\end{gathered}
$$

Степень вырождения энергетических уровней, как и в евклидовом случае, есть $n+1$. Мы видим, что для $U_{1}$ и $U_{2}$ в противоположность евклидову случаю имеет место отличие точных значений $E_{n}$ от квазиклассических из (5) и (6). Собственные функции, соответствуюшие $E_{n}^{(2)}$, имеют вид

$$
\psi_{n, m}(u, \phi)=\left(u^{2}-1\right)^{-\frac{\sigma+n}{2}} u^{\sigma} P_{n, m}^{(2)}\left(u^{2}\right) \exp (i m \phi)
$$

где

$$
P_{n, m}^{(2)}(t)=1+\sum_{j=1}^{k} \frac{(-k)_{j}(-k-|m|)_{j}}{j !\left(-\sigma-n+\frac{1}{2}\right)_{j}}(1-t)^{j}, \quad n=2 k+|m| .
$$

Заметим, что данные собственные функции во всех случаях при $R \rightarrow \infty$ переходят (при надлежашей нормировке) в известные собственные функции соответствуюших задач на пространствах $\mathbf{E}^{2}$ и $\mathbf{E}^{3}$.

Нетрудно видеть, что энергии $E_{n}^{(3)}$ и $E_{n}^{(2)}$ монотонно возрастают с ростом номера уровня $n$, лежашего в указанных пределах. Аналогично случаю $U_{1}$ условия финитности классического движения (2) могут нарушаться при максимальных $n$, причем превышение $E_{n}^{(3)}$ над $U_{2}^{\max }$ опять не превосходит $\frac{\hbar^{2}}{2 R^{2}}$, а превышение $E_{n}^{(2)}$ над $U_{2}^{\max }$ не превосходит $\frac{\hbar^{2}}{8 R^{2}}$.

Данный результат инициирует следуюшую задачу. Пусть $M-$ риманово многообразие бесконечного объема и $-\Delta+U(x)$ - оператор Шредингера на $M \ni x$, причем $U_{\max }=\sup U(x)<\infty$. Требуется найти оценку сверху для $E_{n}-U_{\max }$, где $E_{n}-$ собственные значения данного оператора.

Все указанные выше формулы для собственных значений показывают, что квазиклассический предел эквивалентен евклидову в том смысле, что и при $\hbar \rightarrow 0$, и при $R \rightarrow \infty$ главным членом асимптотики собственных значений является соответствующая классическая формула в евклидовом пространстве.

Автор выражает благодарность за полезные обсуждения данных результатов С. Ю. Доброхотову, В.В. Белову, М.В. Карасеву и А.И. Шаффаревичу, который также обратил внимание автора на работы [2-5]. 


\section{Список литературы}

[1] П.Э. Аппель. Теоретическая механика. М.: ИЛ, 1960.

[2] J. J. Slawianowski. Bull. Acad. pol. sci. Sér. sci. phys. et astron. 1980. V. 28. № 2. P. 99-108.

[3] В. В. Козлов. Вест. МГУ. Сер. 1, Матем. механ. 1994. № 2. С. 28-35.

[4] J. J. Slawianowski, J. Slominski. Bull. Acad. pol. sci. Sér. sci. phys. et astron. 1980. V. 28. № 2. P. 83-94.

[5] V. V. Kozlov, A. O. Harin. Celest. Mech. and Dynam. Astron. 1992. V. 54. P. 393-399.

[6] М. М. Постников. Линейная алгебра. М.: Наука, 1986.

[7] В. И. Арнольд. Математические методы классической механики. М.: Наука, 1989.

[8] В. В. Белов, Е. М. Воробьев. Сборник задач по дополнительным главам математической физики. М.: Высш. школа, 1978.

[9] Б. Саймон, M. Рид. Методы современной математической физики. Т. 2. Гармонический анализ. Самосопряженность. М.: Мир, 1978.

[10] Х. Цикон, Р. Фрезе, В. Кири, Б. Саймон. Операторы Шредингера с приложениями к квантовой механике и глобальной геометрии. М.: Мир, 1990.

[11] Д. Гилбарг, Н. Трудингер. Эллиптические дифференциальные уравнения в частных производных второго порядка. М.: Наука, 1989.

$[12]$ В. В. Голубев. Лекции по аналитической теории дифференциальных уравнений. М.: ГИТТЛ, 1950.

[13] Справочник по специальным функциям. Ред. М. Абрамовиц и И. Стиган. М.: Наука, 1979.

Поступила в редакцию 19.II.1996 г.

\section{A. V. Shchepetilov \\ SOME QUANTUM MECHANICAL PROBLEMS IN LOBACHEVSKY SPACE}

For potentials, being the solutions of Bertrand's problem in Lobachevsky space, quantum mechanical problems are considered. The self-adjointness of the corresponding Schrödinger operators is proved. Energy levels are calculated both from Schrödinger equation and by the Bohr-Sommerfeld method. The effect of quantum binding of classical infinite states is discovered. It is shown that the semiclassical limit is equivalent in some sense to the Euclidean one. 